\title{
Optimum Structural and Manufacturing Design of a Braided Hollow Composite Part
}

\author{
Hossein Ghiasi • Larry Lessard • Damiano Pasini • \\ Maxime Thouin
}

Received: 6 April 2009 / Accepted: 7 October 2009 / Published online: 23 October 2009

(C) Springer Science + Business Media B.V. 2009

\begin{abstract}
Simultaneous material consolidation and shaping, as performed in manufacturing of composite materials, causes a strong interconnection between structural and manufacturing parameters which makes the design process complicated. In this paper, the design of a carbon fiber bicycle stem is examined through the application of a multi-objective optimization method to illustrate the interconnection between structural and manufacturing objectives. To demonstrate the proposed method, a test case dealing with the design of composite part with complex geometry, small size and hollow structure is described. Bladder-assisted Resin Transfer Molding is chosen as the manufacturing method. A finite element model of the stem is created to evaluate the objectives of the structural design, while a simplified $2 \mathrm{D}$ model is used to simulate the flow inside the preform during the injection process. Both models are formulated to take into account the variation of fiber orientation, thickness and fiber volume fraction as a function of braid diameters, injection pressure and bladder pressure. Finally, a multiobjective optimization method, called Normalized Normal Constraint Method, is used to find a set of solutions that simultaneously optimizes weight, filling time and strength. The solution to the problem is a set of optimum designs which represent the Pareto frontier of the problem. Pareto frontier helps to gain insight into the trade-off among objectives, whose presence and importance is confirmed by the numerical results presented in this paper.
\end{abstract}

Keywords Optimum design · Composite material · Resin Transfer Molding ·

Multiobjective optimization $\cdot$ Bicycle stem

\section{Introduction}

Excellent mechanical properties, light weight and appealing appearance have made composite materials a desirable material for many high-performance applications, such as

H. Ghiasi $(\bowtie) \cdot$ L. Lessard $\cdot$ D. Pasini $\cdot$ M. Thouin

Department of Mechanical Engineering, McGill University, Macdonald Engineering Building,

817 Sherbrooke West, Montreal, QC, Canada H3A 2K6

e-mail: hossein.ghiasi@mail.mcgill.ca 
aerospace and sport industries. This demand for composite products has pushed the manufacturers to use high-volume manufacturing methods, such as Resin Transfer Molding (RTM) [1]. RTM is a closed rigid mold process, in which a dry fiber preform is placed between two mold surfaces and the resin is injected through the fibers. The resulting part has a high level of dimensional tolerance and an excellent surface finish. The possibility of manufacturing parts with complex geometries and repeatability of the process make RTM the best choice for many high-performance applications. During the RTM process, material solidification and shaping are performed in one operation, a result of which is a strong interconnection between manufacturing and structural parameters. This coupling effect requires taking into account the manufacturing parameters during the structural design process.

Composite designers usually simplify the design problem by separating the structural design from the process tuning [2] and performing the latter after the structural design is completed. However, researches [2-4] confirm that the approach to the design and optimization of composite materials should be concurrent. In order to demonstrate the importance of these coupling terms, design and optimization of a carbon fiber bicycle stem made by RTM is studied in this research.

Design of a part made by RTM requires not only structural analysis, but also simulation of the flow inside the preform. The flow simulation returns the filling time and finds a proper placement of injection gates and vents that prevent air entrapment and void formation. Here, both structural analysis and flow simulation are performed using simplified, but accurate, numerical models described in the following sections. These models are formulated to be as fast as possible, suitable for numerous calls by an optimization algorithm.

Current literature on coupled structural and manufacturing design of composite parts, almost exclusively, utilizes evolutionary methods (e.g. genetic algorithm) [2-4], despite their low convergence rate and high computational cost. Here on the contrary, a multiobjective optimization scheme in combination with a local-global optimization algorithm is used to find the optimum design that not only leads to a part with high strength, but also minimizes weight and manufacturing time. It has been shown in [5] that this algorithm is faster than an evolutionary method for problems in which the analysis process is time consuming, the number of the solver calls must be limited and only a small number of function evaluations can be performed.

\section{Part and Mold Design}

The part studied here is a bicycle stem, shown in Fig. 1. The stem is a part of the bicycle that connects the handlebar to the fork, through a part called steerer. Its performance is critical, since it is responsible for the control of the bicycle and the comfort of the rider. This section describes the geometry of the part and the corresponding mold.

\subsection{Stem Geometry}

There are different stem-to-steerer tube connection methods available in the market. Among them, the lightest and the most common is used in this research, which is the threadless headset (or aheadset) shown in Fig. 1. The basic design of the stem is similar to the conventional designs, in dimensions and clamping method. The steerer clamp is secured around the steerer tube using two screws. But the handlebar clamp uses four fasteners 
Fig. 1 Stem is a part of the bicycle that connects the handlebar to the fork

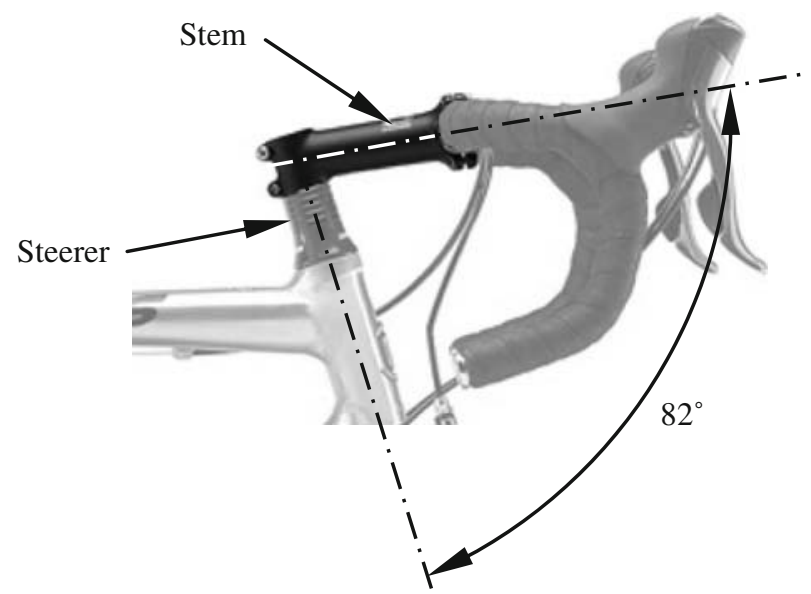

instead of the conventional two, because this configuration is judged to be safer and tends to dissipate the handlebar load over a larger surface. It has four threaded aluminum barrels molded within the stem to fix the cap to the stem body. The wide mouth of the handlebar clamp is designed to create maximum friction on the handlebar, thus it reduces the possibility of slipping and improves stress distribution at the handlebar-clamp interface. The stem body is mostly made of braided carbon fiber sleeves. The braid size must be selected to accommodate the large changes in diameter and still guarantee the expected mechanical properties in critical regions.

The dimensions of the stem are set with an industrial partner in order to be competitive in the market and to fulfill consumer demands. The stem is $120 \mathrm{~mm}$ long and is desired to weigh less than $125 \mathrm{~g}$. The handlebar clamp was made to accommodate a $31.8 \mathrm{~mm}$ handlebar tube, while the steerer tube is $28.6 \mathrm{~mm}$ in diameter. A stem angle of $82^{\circ}$ is used, which is within the market standards and popular among riders.

\subsection{Manufacturing Process and Mold Design}

Resin Transfer Molding (RTM) [1] is selected as the manufacturing process for the production of the stem, considering the small part size, complex geometry, required level of surface finish, desired fast production cycle and the hollow structure. An inflatable bladder is used to produce this hollow component. The combined method, called bladder-assisted $R T M$ [6], is easy from a manufacturing point-of-view and provides a good surface finish and material consolidation.

The selected manufacturing process starts with preparing a preform consisting of several layers of carbon fiber braids. As shown in Fig. 2a, the preform is made by placing the braids of selected diameters on a plastic mandrel, formed as a reduced negative shape of the mold cavity. A thin layer of tackifier is sprayed between each ply to maintain the shape and fiber orientation. The mandrel is then retrieved resulting in a preform which then is placed around an inflatable latex bladder. The preform and the bladder are positioned inside a solid, airtight mold, as shown in Fig. 3. The bladder is inflated draping the fibers to the mold surface. The resin is injected through the injection gate, flowing around the pressurized bladder and leaving the mold through the vent line shown in Fig. 3. After the resin is cured, the part is de-molded and the latex bladder is retrieved. 


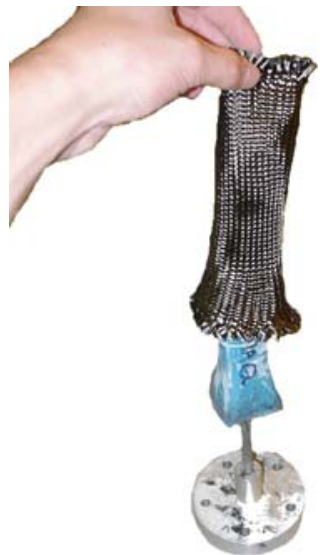

(a)

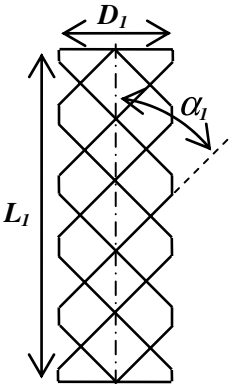

(b)

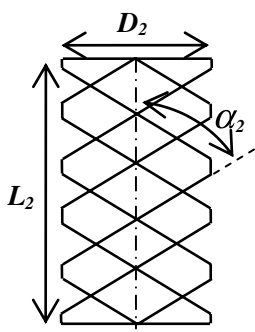

Fig. 2 The preform is made by placing four layers of carbon fiber braid on a plastic mandrel (a) diameter of the braid governs the fiber orientation in the preform (b)

Tooling design for RTM is critical since the quality of the parts and the ease of manufacturing rely mainly on the quality of the tooling [1]. One of the difficulties in designing RTM molds is to avoid air entrapment by strategically placing the injection gates and vents. Gates and vents should be properly located also in order to alleviate de-molding and to leave the shape intact when retrieved. Figure 3 shows the mold designed for the stem body and position of the injection gate and vent within the mold.

Resin selection is performed based on chemical compatibility with the bladder, provided level of surface finish, ease of demolding and availability. Among a few considered resin systems (i.e. Derakane 8084, EPON 862, EPON 9504, Miapoxy), Miapoxy is found to be the best choice for this application [7].

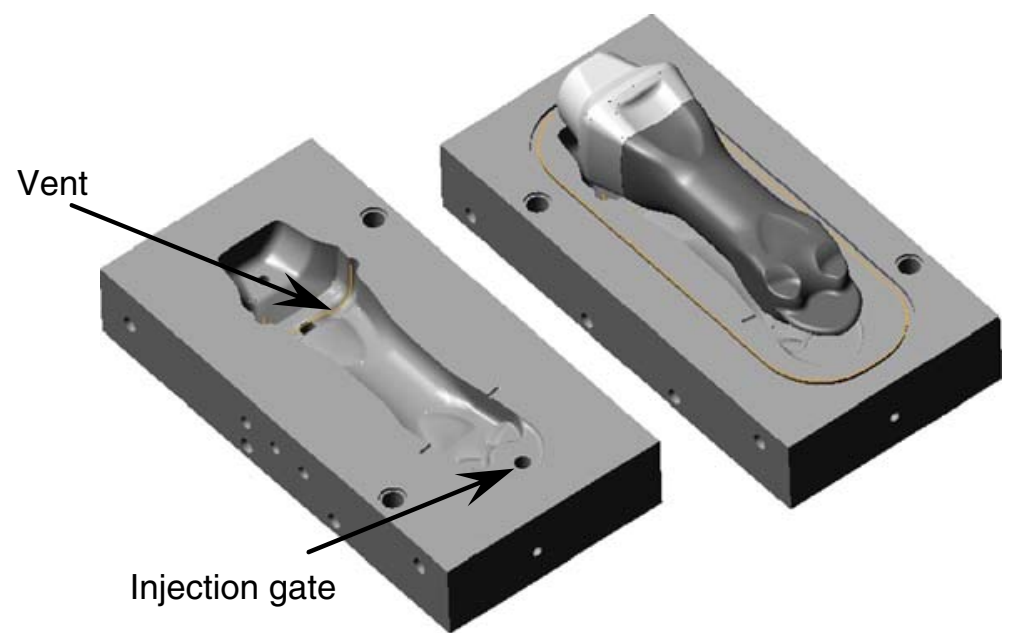

Fig. 3 Mold designed for the production of the stem body 


\section{Structural Analysis}

ANSYS finite element software is employed to analyse the stem structure and to find the failure index that is to be maximized. One of the most challenging issues in structural analysis of the part at hand is the parametric modeling of the local changes in braid diameter and the subsequent variation of the fiber angles, thicknesses and material properties. The finite element model and the critical load cases are described in this section.

\subsection{Variations in Material Properties}

The preform is made of continuous braided carbon fiber sleeves, which have the particularity of varying fiber angle and thickness when the diameter is changed (demonstrated in Fig. 2b). Because of the changes in fiber orientation, the braid diameter has a major effect on material properties and preform permeability. In order to take into account the changes in braid diameter and material properties, the stem is separated into six different regions with different material properties (shown in Fig. 4). The sections are chosen such that the fiber angles would be accurate to $\pm 5^{\circ}$. The mechanical properties at each region are calculated as functions of braid diameter and applied pressure to the preform.

Fiber orientation and thickness of the preform is provided by the manufacturer as a function of the braid diameter to its nominal diameter. The nominal diameter $\left(d_{n}\right)$, nominal fiber volume fraction $\left(v_{f n}\right)$ and nominal thickness $\left(t_{n}\right)$ of the braid are the parameters provided by the manufacturer and are measured when the fibers are oriented at \pm 45 degrees under $200 \mathrm{kPa}$ pressure at room temperature. Figure 5 shows the fiber orientation and thickness of the preform as functions of relative braid diameter. This chart is generated by interpolating the data provided by the manufacturer.

Figure 6 shows the fiber volume fraction versus pressure applied to the preform. This pressure is equal to the difference between the bladder pressure and the resin injection pressure. The higher the bladder pressure, the higher the fiber volume fraction. The fiber volume fraction found from this chart is used to calculate the mechanical properties of the composite material, which are a weighted sum of the properties of the fibers and the resin.

Another important property affected by the changes in braid diameter is the preform permeability. Variation of this parameter depends on two other parameters, namely, the fiber volume fraction and the fiber orientation. In order to characterize this property, samples are

Fig. 4 The finite element model highlighting regions with different material properties

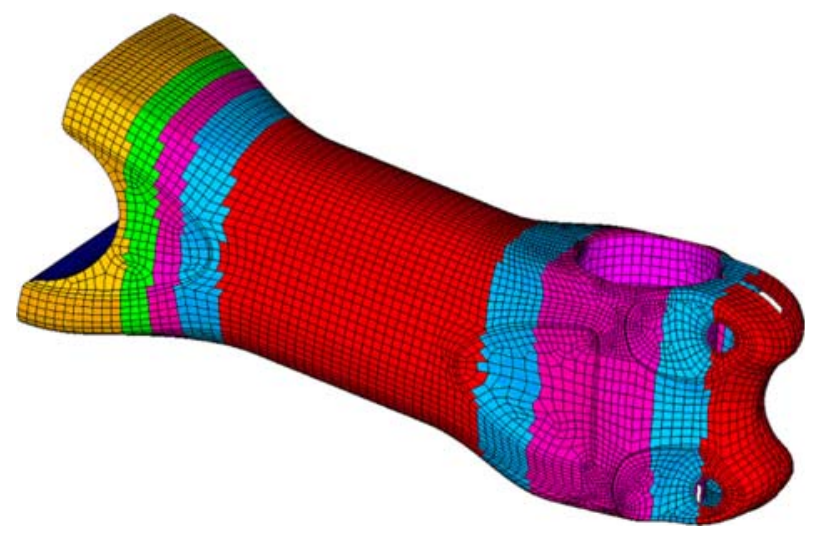


Fig. 5 Fiber orientation and thickness as functions of relative diameter

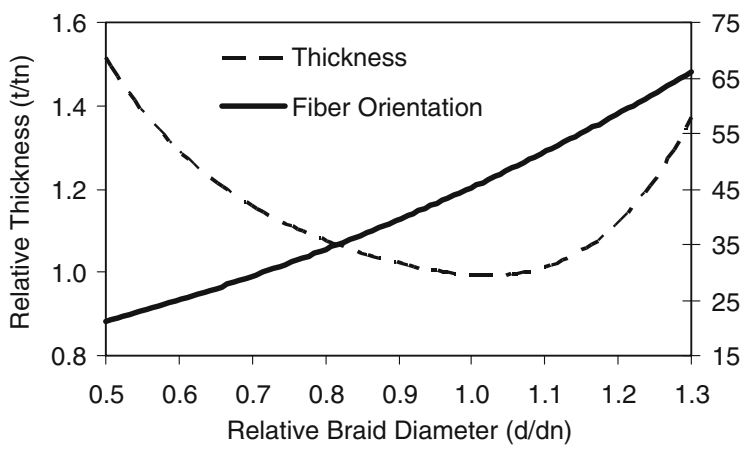

made with fibers oriented at $30^{\circ}, 45^{\circ}$, and $60^{\circ}$. Each sample is tested at least with three different fiber volume fractions. The permeability is interpolated as a power function, with the results presented in Figure 7.

\subsection{Loading}

The stem contributes to the ease of handling and the position and comfort of the rider, and as a result, it bears a significant amount of load $[8,9]$. The loading is difficult to model because of the clamping pre-load and unreliability of data on the extreme load cases. The clamping loads at the steerer clamp are modelled by applying a radial constraint on the inner surface of the clamp and a pre-load of $2000 \mathrm{~N}$ on each of the two fasteners at this clamp. The pre-load on the handlebar clamp is different, because a radial constraint, as it is applied to the steerer clamp, prevents this part from bending and rotating under bending and torsional loads. Thus, the pre-load in the handlebar clamp is modeled by a uniform pressure applied to its inner surface. The amount of pressure is selected to equilibrate the $1400 \mathrm{~N}$ load applied on each of the four fasteners at this clamp. In addition to the clamping load, the critical riding loads are also applied as defined by Van Der Aa [10], an out-of-phase loading of $+164 /-147 \mathrm{~N}$ in the vertical direction applied at the end locations of a standard $44 \mathrm{~cm}$ handlebar. It is also considered that the rider applies $9 \mathrm{~N}$ in the horizontal direction at each hand location.

Fig. 6 Variation of fiber volume fraction as a function of pressure applied to the preform (i.e. bladder pressure minus injection pressure)

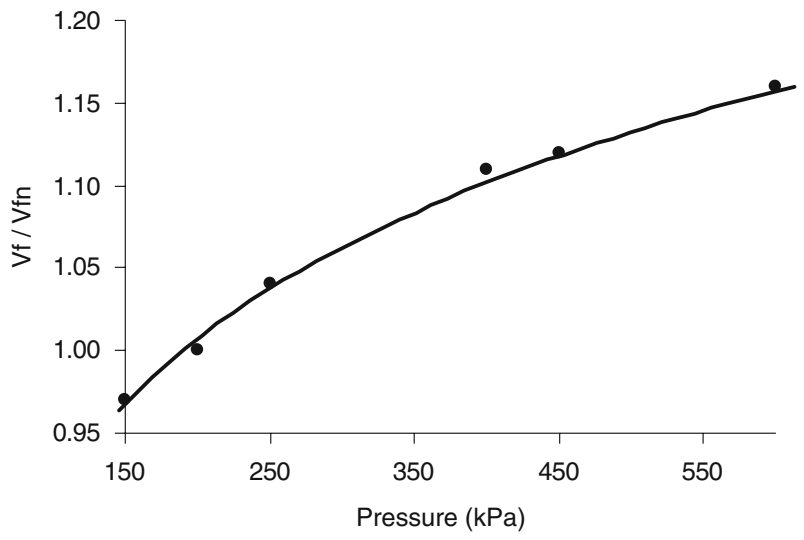


Fig. 7 Preform permeability as a function of fiber volume fraction and fiber orientation

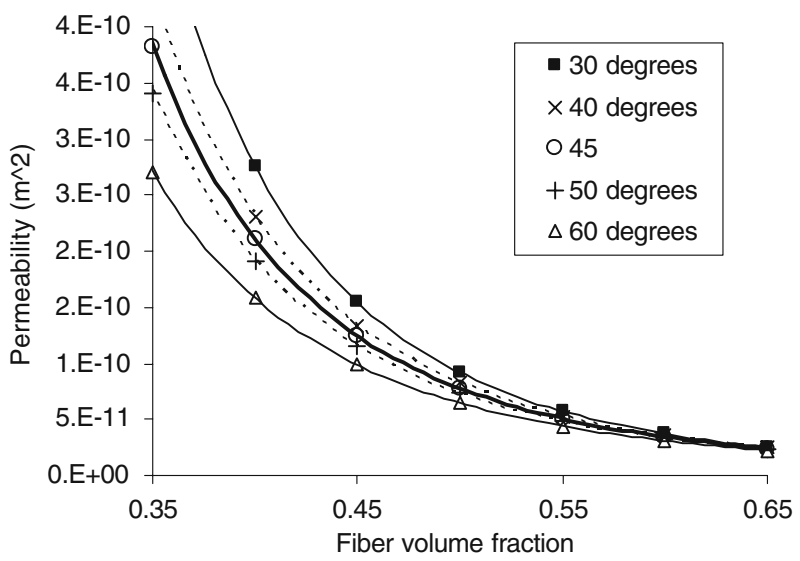

\subsection{Failure Analysis}

The failure analysis is performed in ANSYS using Tsai-Wu failure criterion [11]. The finite element model and the applied boundary conditions are presented in Fig. 8. The inverse strength ratio [11] is used as an objective function to be minimized during the optimization process. In order to perform the failure analysis, a parametric APDL code is generated. The APDL code reads the design variables (i.e. braid diameter, bladder pressure and injection pressure) from an input file and calculates the fiber orientation and material properties corresponding to each element. Then the finite element analysis is performed and the maximum inverse strength ratio is returned to the optimization process using a text file.

\section{Process Simulation}

In order to obtain a good result in the RTM process, injection pressure, bladder pressure and injection temperature must be optimally selected and controlled. Here, the injection and cure temperature are set to the ones provided by the resin manufacturer. The bladder and injection pressures are found to have a significant effect on all the objectives. The effect of these

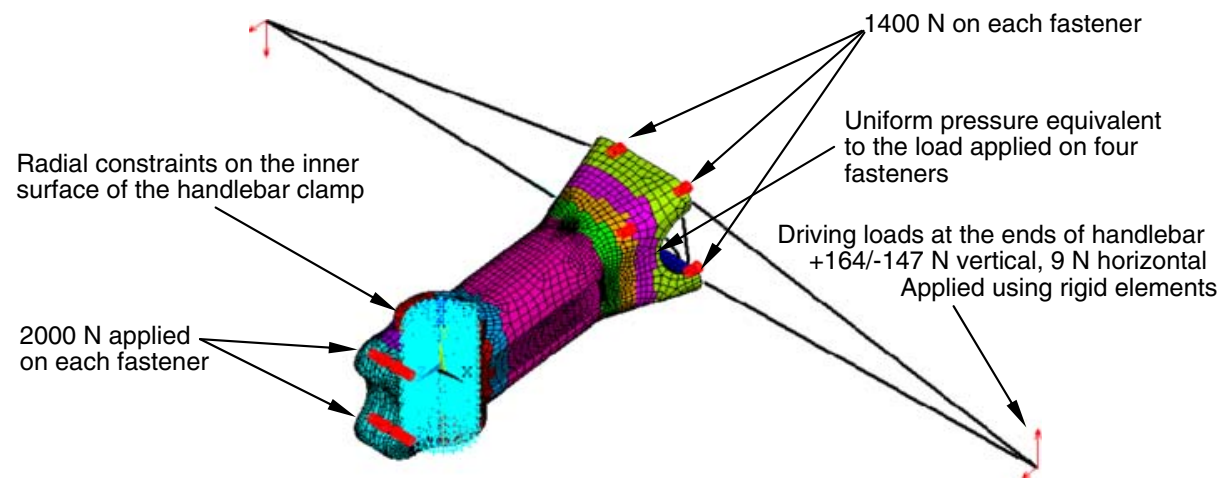

Fig. 8 Simplified finite element model of the stem showing the applied boundary conditions, loads and regions with different materials properties 
parameters on strength is taken into account by considering the changes in fiber volume fraction, as discussed in previous sections. The quality of the surface finish is also significantly affected by these two parameters. A better surface finish is achieved if a higher bladder pressure is applied; however, a good surface finish can also be achieved by increasing the bladder pressure just after the injection is clamped. Therefore, during the injection process, the bladder pressure only needs to be as high as required to ensure the resin flows through the preform and that complete wetting is achieved. This goal is achieved by forcing the bladder pressure to be at least $50 \mathrm{kPa}$ more than the injection pressure. Finally, in order to find the injection time, simulation of the resin flow inside the pressurized preform is required.

\subsection{Flow Simulation Inside the Mold}

Performing a 3D simulation of the flow inside the preform is a time consuming process and requires a specialized finite element package; however, considering that during the injection process, resin flows through a thin preform pressed against the inner surface of the mold cavity, the flow can be approximated by a two-dimensional flow. In order to perform a two dimensional flow simulation, a 2D model of the expanded shape of half of the stem is created, as shown in Fig. 9. The width of the 2D field in each section is set equal to half the perimeter of the actual part at that section. Figure 10 shows the perimeter of a few cross sections of the stem, which are used to create the 2D model in Fig. 9. In order to simulate the flow inside this 2D field, the method developed by Boccard et al. [12] is employed.

This method calculates the filling time for a thin flat preform with a constant and isotropic permeability all over the field. For the problem at hand, the permeability of the preform varies from one point to another and, more importantly, the preform does not have an isotropic permeability. The permeability matrix is calculated for seven different regions within the 2D model, as shown in Fig. 9. The transformation by Chan et al. [13] is used to transform each region into an equivalent region with a constant, isotropic permeability. The flow inside this equivalent $2 \mathrm{D}$ field is then used to find the injection time.

Boccard's method [12] calculates the time required for the resin to reach each point on the perimeter of the $2 \mathrm{D}$ field. This method is applied to find the filling time for 22 selected points around the perimeter of the 2D model, as shown in Fig. 9. The time required for the resin to reach these points is plotted in Fig. 11 (left). Time varies with the braid diameter, injection pressure and bladder pressure, but the overall shape of the graph remains the same. The filling time plotted in Fig. 11 (left) shows that points 9 and 15 are the last points in the part reached by the resin. This prediction is in agreement with the observation made in experiment, an example of which is shown in Fig. 11 (right). This figure shows that a

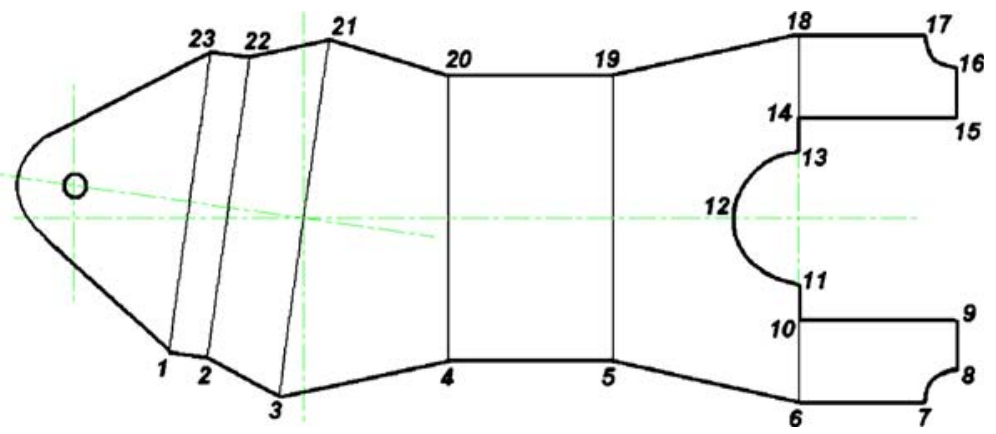

Fig. 9 The two-dimensional model of half of the stem is used to simulate the flow inside the preform 

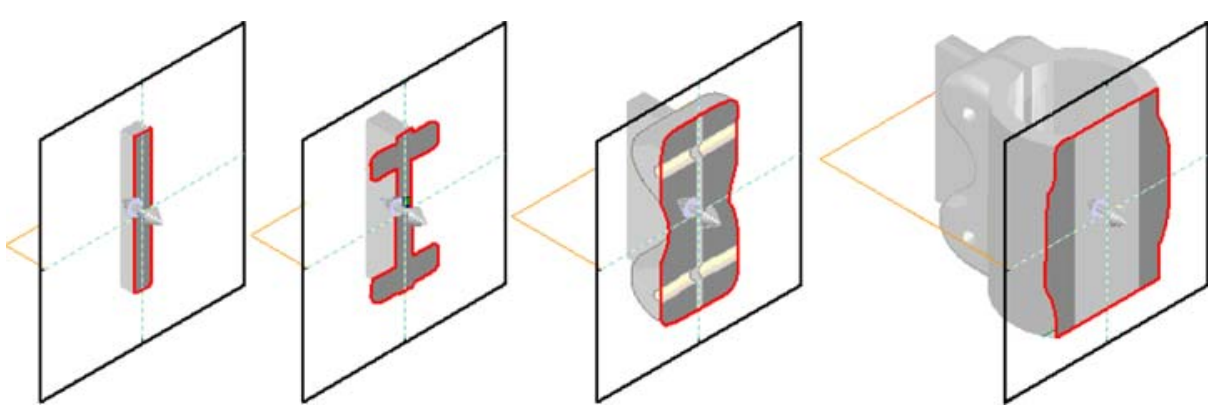

Fig. 10 The perimeter of the part in a few cross sections, as used to generate the simplified 2D model

void is formed at point (9) as predicted by the numerical flow simulation. The filling time measured for an experimental design is around $10 \mathrm{~min}$, which is very close to the filling time calculated with this method (i.e. $582 \mathrm{~s}$ ).

\section{Optimization Problem}

The optimization problem consists of finding a design that provides the minimum weight and mold filling time as well as the maximum strength for a stem made of four carbon fiber braids. Braid diameters, injection pressure and bladder pressure are the design variables. The optimization problem is formulated as follows:

$$
\begin{gathered}
\min _{d_{i}, P_{\text {inj }}, P_{\text {bladder }}}\left\{\begin{array}{l}
W\left(d_{i}, P_{\text {inj }}, P_{\text {bladder }}\right) \\
T\left(d_{i}, P_{\text {inj }}, P_{\text {bladder }}\right) ; i=1, \ldots, 4 \\
S\left(\left(d_{i}, P_{\text {inj }}, P_{\text {bladder }}\right)\right.
\end{array}\right. \\
\text { s.t }\left\{\begin{array}{l}
38.1 \mathrm{~mm}<d_{i}<63.5 \mathrm{~mm} ; i=1, \ldots, 4 \\
100 \mathrm{kPa}<P_{\text {inj }}<450 \mathrm{kPa} \\
150 \mathrm{kPa}<P_{\text {bladder }}<500 \mathrm{kPa} \\
P_{\text {bladder }}-P_{\text {inj }} \geq 50 \mathrm{kPa}
\end{array}\right.
\end{gathered}
$$
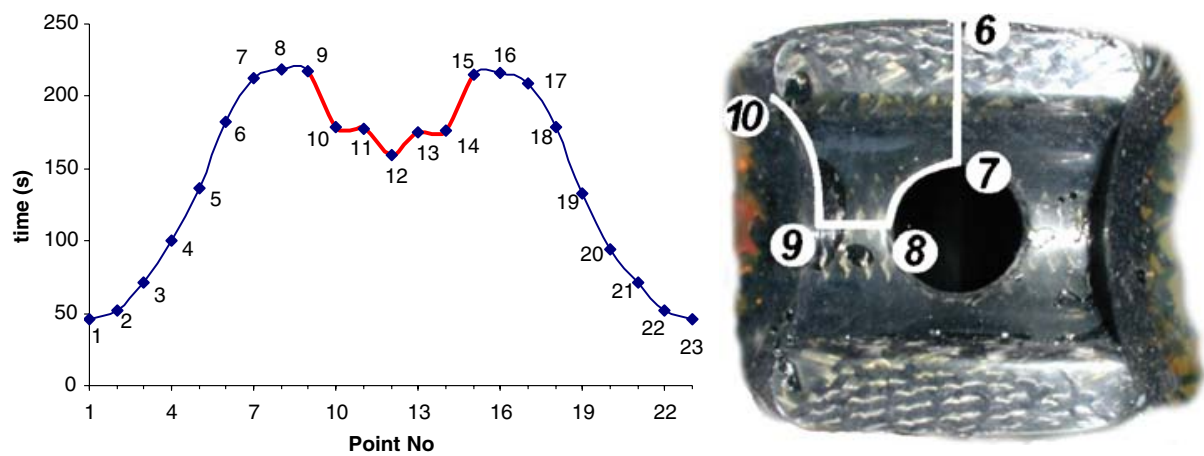

Fig. 11 The time required for the resin to reach each point on the boundary of the preform (left) The experimental results confirm the formation of void at point 9 as predicted by the numerical simulation (right) 
where $W, T$ and $S$ in this equation represent weight, filling time and the inverse strength ratio, respectively. $d_{i}$ is the braid diameter, and $P_{i n j}$ and $P_{\text {bladder }}$ stand for the injection pressure and the bladder pressure. The flowchart in Fig. 12 shows the interconnection between the design variables and the objectives. As explained in the preceding sections and also shown in Fig. 12, manufacturing and structural parameters are interconnected and it is not possible to perform a separate optimization that first finds the best structure by changing the braid diameters and then selects the optimum pressures that provide the fastest manufacturing process. Instead, all the objectives must be considered simultaneously.

\subsection{Optimization Algorithm}

Simultaneous optimization of structural and manufacturing objectives requires a multiobjective optimization (MOO) procedure. A comprehensive review of these methods can be found in [14-16]. The current literature in MOO of composite materials reveals mainly the use of non-generating methods. Although popular for their simplicity and for being generally less time consuming than generating methods, the drawback associated with nongenerating methods is that they return only one optimum solution derived by user preferences. The Method of weighted-sum, which is the most common MOO method, belongs to this group. Examples of applications of this method in stacking sequence design of composite materials can be found in [17-20]. Another common non-generating method is called the $\varepsilon$-constraint method. It optimizes one criterion while the others are constrained to user-defined limits $[2,21,22]$. Another common non-generating method is the referencepoint method, in which a user-defined measure (e.g. Euclidian distance) based on a userdefined reference point is minimized [23, 24]. The min-max strategy is another example of these methods, in which the most critical objective (e.g. the maximum stress [25]) is minimized. The limitation is that this method requires the objectives to be of the same nature (i.e. all the objectives should be either stress minimization or weight minimization). Appropriate selection of weighting factors, constraints and reference point has a major effect on the final solution obtained by these methods. Therefore, finding an appropriate solution requires a priori insight into the problem, which is often not available.

There are also attempts in using generating methods for the design of a composite part. Most of generating algorithms use a population-based stochastic method that requires numerous function evaluations. Examples of these methods being used in composite design are Improved Non-dominated Sorting Genetic Algorithm (NSGA-II) [26, 27] and Vector Evaluated Particle Swarm Optimization (VEPSO) [28]. Generating methods do not require any user-defined preferences, but they usually need a great deal of computation.

Composite design problems have the particularity of having a time consuming function evaluation process, which usually involves performing several finite element analyses. Therefore, in many cases, it may not be possible or desirable to perform as many function evaluations as required for a population-based method to converge. For these situations, a

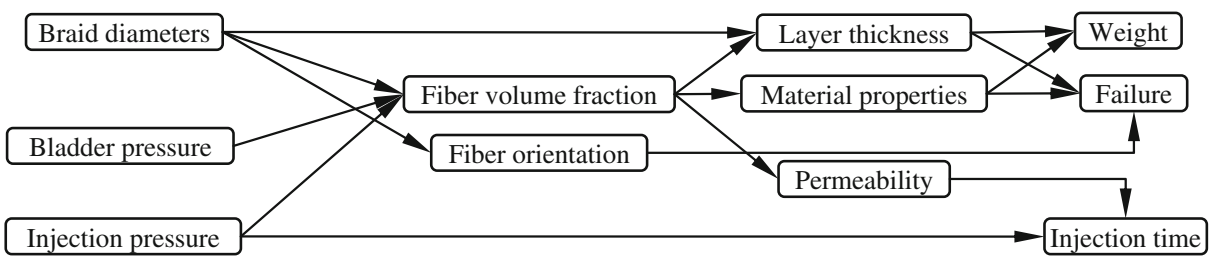

Fig. 12 Interconnections among design variables and objectives 
combination of a local single-objective optimization technique and a MOO approach, called $\mathrm{NNCM}$, is found to be more efficient than an evolutionary method [5].

Normalized Normal Constraint Method (NNCM) [29] is a MOO method that transforms a multiobjective problem into several single-objective optimization problems; each corresponds to one optimum solution. All solutions together represent a figure called Pareto frontier, which for the stem design problem, is a 3D surface as shown in Fig. 13.

The NNCM procedure for a problem with two objectives can be found in [29]. Here, the NNCM is applied to a problem with three objectives. The optimization process includes solving three single objective problems providing the optimum value of the three objectives, separately. These solutions, called anchor points, are used to normalize the criterion space. The criterion space for this problem is a 3D space representing the three objectives. The criterion space is normalized so that the minimum of each objective is located at the origin and the maximum takes the unit value (see Fig. 13-left). NNCM then restrains the feasible region by introducing constraints normal to the plane passing through the anchor points (Fig. 13-right). Applying each set of constraints to the original problem leads to a single objective problem, whose solution is a point on the Pareto frontier.

Here, the set of single objective optimization problems generated by NNCM are solved using a local-global optimization method based on probabilistic restart of Nelder-Mead (N-M) method. The method is called Globalized Bounded Nelder-Mead method (GBNM) $[30,31]$ and uses a probability function that repeatedly restarts the N-M local search. The probabilistic restart permits the local search to initiate a new search from points different from those already explored. Figure 14 shows the general flowchart of the optimization method, which includes N-M at the innermost loop, probabilistic restart by GBNM in the intermediate level, and finally the NNCM that repeats the entire cycle to find different points on the Pareto frontier.

\section{Results and Discussion}

For the stem design problem, finding anchor points corresponds to performing three separate single-objective optimizations, each of which optimizes only one of the desired objectives (i.e. strength, filling time or weight). Other than anchor points, four points on the Pareto frontier are sought. Three of these points correspond to the designs that
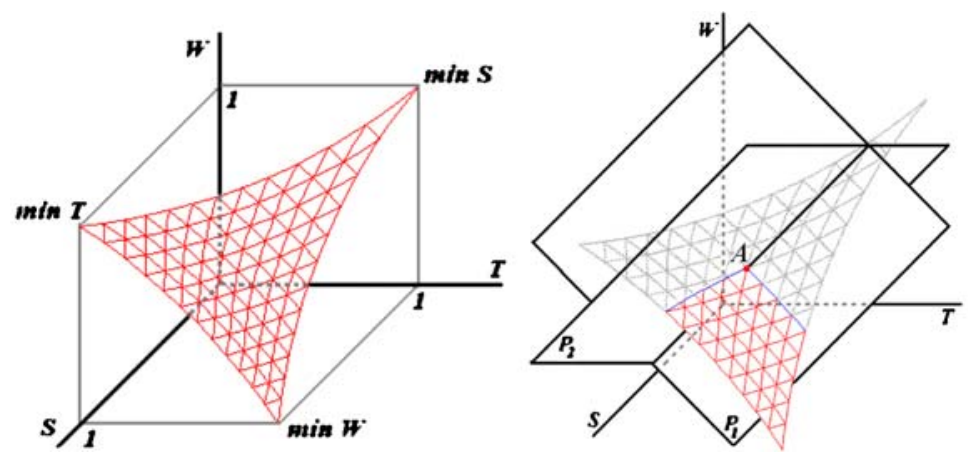

$\mathrm{S}$ : inverse Tsai-Wu strength ratio

$\mathrm{T}$ : filling time

W: weight

Fig. 13 The optimum points for a MOO problem present a figure called Pareto frontier. Pareto frontier for the stem problem is a $3 \mathrm{D}$ surface (left). If the original MOO problem is constrained with planes normal to the plane of $\min S, \min T, \min W$, (right), minimizing $\mathrm{S}$ returns a point on the Pareto frontier, like A 


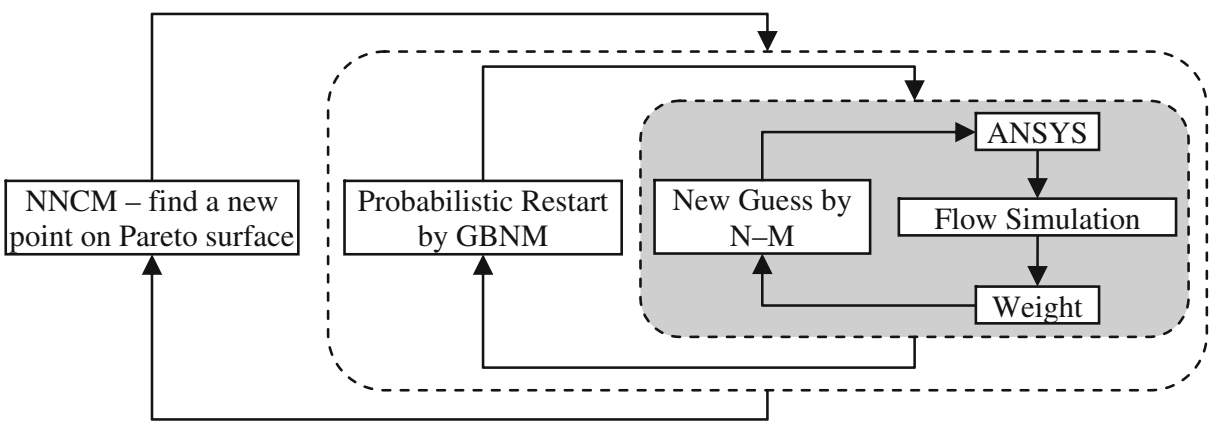

Fig. 14 Flowchart of the optimization algorithm

simultaneously take into account a pair of these objectives and the fourth one correspond to the case that all three objectives are simultaneously optimized. One should note that the Pareto front is not necessarily restricted to the points within the triangle formed by connecting three anchor points, here these four points are selected to give an approximate image of the trade-off among objectives. The optimization process for each point is conducted for up to 500 iterations of the N-M method (i.e. almost equal to performing 1000 function evaluations) and the results are shown in Table 1. Minimizing weight and filling time are not time consuming, since the corresponding analysis is performed in a MATLAB code, but the structural problem that needs ANSYS finite element analysis is a time consuming process. Performing 500 iterations of the N-M method that includes a structural analysis approximately takes five hours on a machine with an Intel-Pentium $4 \mathrm{CPU}$ (3.2GHZ) and $2 \mathrm{~GB}$ of RAM.

Figure 15 shows the location of the optimum solutions in a schematic form (a) and as they are found by the optimization method (b). This figure along with Table 1, gives a designer the opportunity to see the trade-off among the objectives. For instance, it shows that the simultaneous optimization of strength and weight (solution marked by $S W$ ) finds a design that is stronger than " $\min W$ " but with the consequence of being $41 \%$ heavier. The same design in comparison with " $\min S$ " is $10 \%$ lighter with the penalty of being less strong. A similar trade-off exists between the multiobjective design point "STW" and the

Table 1 Solutions of the separate and simultaneous optimization problems

\begin{tabular}{lllllllll}
\hline Design Parameters & $\min S$ & $\min T$ & $\min W$ & $S W$ & $T W$ & $S T$ & $S T W$ & $\operatorname{Exp}^{\mathrm{a}}$ \\
\hline Braid diameter 1 (mm) & 63.5 & 63.5 & 48.8 & 50.6 & 41.4 & 40.1 & 63.3 & 63.5 \\
Braid diameter 2 (mm) & 38.1 & 63.5 & 47.5 & 38.2 & 44.4 & 43.7 & 41.5 & 50.8 \\
Braid diameter 3 (mm) & 38.1 & 63.5 & 48.7 & 49.8 & 42.5 & 40.1 & 61.1 & 50.8 \\
Braid diameter 4 (mm) & 63.5 & 63.5 & 48.9 & 43.7 & 40.3 & 40.1 & 38.3 & 63.5 \\
Injection pressure (kPa) & 427 & 450 & 100 & 102 & 103 & 100 & 166 & 275 \\
Bladder pressure (kPa) & 482 & 500 & 500 & 153 & 284 & 176 & 230 & 450 \\
Inverse Strength Ratio & 1.424 & 2.089 & 3.379 & 1.946 & 2.984 & 2.555 & 1.839 & 2.74 \\
Filling time (s) & 104 & 43 & 4972 & 426 & 2506 & 967 & 312 & 582 \\
Weight (g) & 93.4 & 83.6 & 59.4 & 83.9 & 71.5 & 83.5 & 85.6 & 68.2
\end{tabular}

${ }^{a}$ this point is found by experiment and trying different combination of design parameters 
anchor points. The last point in Table 1, labelled as "Exp", is the best design found by experiment after trying different combinations of design parameters [7]. As shown in Fig. 15, the experimental design is almost located on the Pareto frontier found by the optimization method.

Figure $15 \mathrm{~b}$ shows all the optimum solutions found during the optimization process. It shows that the Pareto surface for the problem at hand has a tendency toward the minimum filling time and the maximum strength (i.e. minimum inverse strength ratio). In other words, the trade-off between weight and time or weight and strength is stronger than the trade-off between time and strength, which is reasonable. Weight reduction is achieved by reducing the part thickness, while the amount of fiber is almost constant (because the number of braids are kept constant at four layers); therefore, weight reduction does not lead to a significant deterioration of the strength, but increases the filling time significantly, since the compressed preform has a higher fiber volume fraction and is less permeable. The multiobjective optimization gives such insight into the problem and leaves the designer with a general view of all the possible solutions. Now it is the designer's choice to pick the best solution among possible optimum solutions.

\section{Conclusions}

This paper has examined the simultaneous structural and manufacturing design of a composite part. The design of a carbon fiber bicycle stem is used as a practical example to demonstrate the level of interconnection between structural and manufacturing parameters. A parametric finite element model of the part is created in ANSYS that considers variation of structural properties as a function of structural and manufacturing parameters. In order to perform a fast but reliable flow simulation, a simplified 2D model of the flow field inside the part is created. Finally, a multi-objective optimization algorithm, called Normalized Normal Constraint Method (NNCM) is applied to solve the corresponding multiobjective

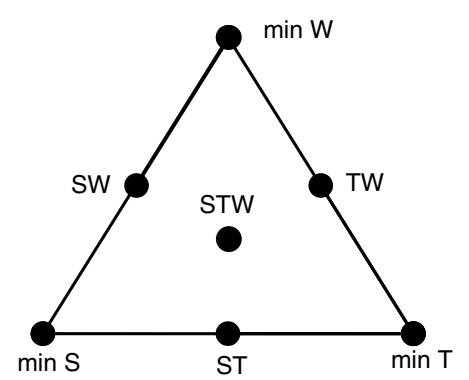

(a)

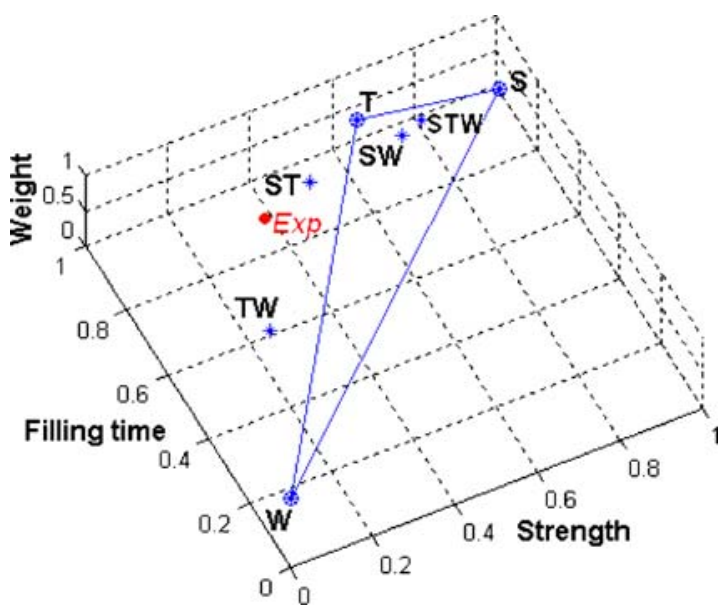

(b)

Fig. 15 Locations of separate and simultaneous designs with respect to three objectives. (a) schematic position of the solutions (b) the actual results obtained for the stem design problem. All objectives are normalized such that the best value is " 1 " and the worst is " 0 " 
optimization problem. The result is a set of optimum solutions that represents the Pareto frontier of the problem, which in this case is a 3D surface. Pareto frontier helps the designer to understand the trade-off among objectives. The results show that such a conflict between weight, strength and manufacturing cost (represented by injection time) exists in this particular example and this algorithm is able to show this trade-off in a useful way.

\section{References}

1. Potter, K.: Resin transfer molding, London, New York, Chapman \& Hall (1997)

2. Le Riche, R., Saouab, A., Breard, J.: Coupled compression RTM and composite layup optimization. Comp. Sc. and Tech. 63(15), 2277-2287 (2003)

3. Park, C.H., Lee, W., Han, W.S., Vautrin, A.: Weight minimization of composite laminated plates with multiple constraints. Compo. Sc. Tech. 63(7), 1015-1026 (2003)

4. Park, C.H., Lee, W., Han, W.S., Vautrin, A.: Multiconstraint optimization of composite structures manufactured by resin transfer molding process. J. Compo. Mat. 39(4), 347-374 (2005)

5. Ghiasi, H., Pasini, P., Lessard, L.: Pareto frontier for simultaneous structural and manufacturing optimization of a composite part. Struc. Multidisc. Optim. (2009). doi:10.1007/s0015800903664

6. Lehmann, U., Michaeli, W.: Improved processing of resin transfer molding for the production of hollow parts with inflatable bladders. In: Proceedings of the 42nd International SAMPE Symposium and Exhibition 42(1): 13-23 (1997)

7. Thouin, M.: Design of a carbon fiber bicycle stem using an internal bladder and resin transfer molding. M.Eng. Thesis, McGill University (2004)

8. Lessard, L., Nemes, J., Lizotte, P.: Utilization of FEA in the design of composite bicycle frames. J. Composites 26(1), 72-74 (1995)

9. Lizotte, P.: Stress analysis and fabrication of composite monocoque bicycle frames. M.Eng. Thesis, McGill University (1996)

10. Van der Aa, H.C.E.: Re-design and manufacturing of a composite monocoque bicycle frame. Joint Technical Report, McGill University and T.U. Eindhoven, Netherlands (1997)

11. ANSYS theory reference, Release 11.0: documentation for ANSYS, ANSYS, Inc.

12. Boccard, A., Lee, W.I., Springer, G.S.: Model for determining the vent locations and the fill time of resin transfer molds. J. Compo. Mat. 29, 306-333 (1995)

13. Chan, A.W., Larive, D.E., Morgan, R.J.: Anisotropic permeability of fiber preforms: constant flow rate measurement. J Compo. Mat. 27(10), 996-1008 (1993)

14. Miettinen, K.: Nonlinear multiobjective optimization. Kluwer Academic Publishers, Boston (1999)

15. Deb, K.: Multi-objective optimization using evolutionary algorithms. John Wiley \& Sons, LTD, Chichester, New York, Weinheim, Brisbane, Singapore, Toronto (2001)

16. Marler, R.T., Arora, J.S.: Survey of multiobjective optimization methods. Struct. Multidisc. Optim. 26, 369-395 (2004)

17. Walker, M., Reiss, T., Adali, S.: Multiobjective design of laminated cylindrical shells for maximum torsional and axial buckling loads. Compu. Struc. 62(2), 237-242 (1997)

18. Deka, D.J., Sandeep, G., Chakraborty, D., Dutta, A.: Multiobjective optimization of laminated composites using finite element method and genetic algorithm. J Reinf. Plast. Compo. 24(3), 273-285 (2005)

19. Mohan Rao, A.R., Arvind, N.: A scatter search algorithm for stacking sequence optimisation of laminate composites. Compo. Struc 70, 383-402 (2005)

20. Abouhamze, M., Shakeri, M.: Multi-objective stacking sequence optimization of laminated cylindrical panels using a genetic algorithm and neural networks. Compo. Struc. 81(2), 253-263 (2007)

21. Wang, B.P., Costin, D.P.: Optimum design of a composite structure with three types of manufacturing constraints. AIAA J. 30(6), 1667-1669 (1992)

22. Henderson, J.L., Gurdal, Z., Loos, A.C.: Combined structural and manufacturing optimization of stiffened composite panels. J of Aircraft 36(1), 246-254 (1999)

23. Saravanos, D.A., Chamis, C.C.: Multiobjective shape and material optimization of composite structures including damping. AIAA 30(3), 805-813 (1992)

24. Kere, P., Lento, J.: Design optimization of laminated composite structures using distributed grid resources. Compo. Struc. 71(3-4), 435-438 (2005)

25. Suresh, S., Sujit, P.B., Rao, A.K.: Particle swarm optimization approach for multi-objective composite box-beam design. Compo. Struc. 81(4), 598-605 (2007) 
26. Deb, K., Pratap, A., Agarwal, S., Meyarivan, T.: A fast and elitist multiobjective genetic algorithm: NSGA-II. IEEE Trans. Evol. Comput. 6(2), 182-197 (2002)

27. Pelletier, J.L., Vel, S.S.: Multi-objective optimization of fiber reinforced composite laminates for strength, stiffness and minimal mass. Compu. Struc. 84, 2065-2080 (2006)

28. Omkar, S.N., Mudigere, D., Naik, G.N., Gopalakrishnan, S.: Vector evaluated particle swarm optimization (VEPSO) for multi-objective design optimization of composite structures. Compu. Struc. 86, $1-14$ (2008)

29. Messac, A., Ismail-Yahaya, A., Mattson, C.A.: The normalized normal constraint, method for generating the Pareto frontier. Struc. Multidisc. Optim. 25(2), 86-98 (2003)

30. Luersen, M.A., Le Riche, R.: Globalized Nelder-Mead method for engineering optimization. Compu. Struc. 82, 2251-2260 (2004)

31. Ghiasi, H., Pasini, D., Lessard, L.: Constrained globalized Nelder-Mead method for simultaneous structural and manufacturing optimization of a composite bracket. J. Compo. Mat. 42(7), 717-736 (2008) 